\title{
Kernos
}

Revue internationale et pluridisciplinaire de religion grecque antique

19 | 2006

Varia

\section{Les mentions généalogiques chez Pindare}

\section{Emilio Suárez de la Torre}

\section{OpenEdition \\ Journals}

Édition électronique

URL : https://journals.openedition.org/kernos/435

DOI : $10.4000 /$ kernos.435

ISSN : 2034-7871

\section{Éditeur}

Centre international d'étude de la religion grecque antique

\section{Édition imprimée}

Date de publication : 1 janvier 2006

Pagination : $97-111$

ISSN : 0776-3824

\section{Référence électronique}

Emilio Suárez de la Torre, « Les mentions généalogiques chez Pindare », Kernos [En ligne], 19 | 2006, mis en ligne le 07 septembre 2011, consulté le 24 août 2022. URL : http://journals.openedition.org/ kernos/435; DOI : https://doi.org/10.4000/kernos.435 


\title{
Les mentions généalogiques chez Pindare
}

\begin{abstract}
Résumé : Analyse de la fonction et caracteristiques de la liaison entre la victoire sportive et le passé 'mythique' par l'intermédiaire du lien généalogique avec un dieu et/ou un héros. Examen des exemples suivants: Olympique 2, Isthmique 3, Ol. 6, Ol. 9, Ol. 7, Pythique 4, Pyth. 5, Néméenne 11. La mention généalogique renforce le moment de gloire de la victoire (conçue comme conséquence des origines), ajoute parfois une perspective religieuse, génère une dimension spatiale (et à la fois temporelle et historique), contribue à l'organisation du poème et devient un recours encomiastique très efficace.
\end{abstract}

Abstract: Myth and Genealogy in Pindar. An analysis of the function and characteristics of the link between the victory in the games and the 'mythical' past, through a genealogical relationship with a god and/or hero. An examination of the following poems: Olympian 2, Isthmian 3, Ol. 6, 9 and 7, Pythian 4 and 5, Nemean 11. The genealogical reference reinforces the glory of the victory (conceived as a consequence of the prestigious origins of the lineage), adds sometimes a religious perspective, creates a spatial dimension (and simultaneously a temporal and a historical one), contributes to the organisation of the poem and becomes a very efficient encomiastic resource.

\section{Introduction}

Cette étude vise à analyser la fonction et caractéristiques de la liaison entre la victoire sportive et le passé mythique par l’intermédiaire du lien généalogique avec un dieu et/ou un héros. On se limitera donc aux poèmes dont le récit mythique fait allusion expressis verbis à l'existence de ce lien, sans prêter attention à ceux où la lignée généalogique pourrait être déduite de façon générale depuis un premier niveau de fondation mythique plus ou moins ancestral $^{1}$. Les cas de descendance divine et/ou héroïque, pleinement identifiée et individualisée, du génos du vainqueur seront seuls pris en compte.

Le terme 'mythique' est employé pour des raisons purement pratiques, car pour les Grecs (et surtout à propos des questions généalogiques) il ne s'agit pas d'un niveau qu'on pourrait différencier radicalement de celui de l'histoire (sans le devenir nécessairement) et d'un passé parfaitement repérable tout au long du cours du temps. Dans ce sens il faudrait renvoyer, d'un côté, à l'analyse (brève, mais bien substantielle) de Huxley $^{2}$ et, d'un autre, aux perspectives ouvertes par les travaux de Calame $e^{3}$. Dans tous les exemples commentés on aperçoit une conviction profonde de la persistance à travers

\footnotetext{
${ }^{1}$ C'est le cas, par exemple, des références à la généalogie des Éacides dans les odes pour des vainqueurs d'Égine.

${ }^{2}$ G. HuXLEY, Pindar's Vision of the Past, Belfast, 1975.

${ }^{3}$ C. CALAME, «'Mythe' et 'rite' en Grèce: des catégories indigènes ? ", Kernos 4 (1991), p. 179204; id., Mythe et histoire dans l'Antiquité grecque. La création symbolique d'une colonie, Lausanne, Payot, 1996.
} 
les générations des traits définiteurs (au sens large) d'un génos, grâce au miracle de la transmission du sperma qui enferme ces qualités. Ces traits composent un tableau qui apparaît au niveau poétique dans les nombreuses images végétales et qui est construit autour d'un réseau lexico-sémantique bien connu ${ }^{4}$.

\section{Généalogie, mythe et eschatologie : Olympique 2 (476) et Istbmique 3 (c. 476/473)}

Cette ode exceptionnelle par tant de motifs renferme plus d'une mention de l'importance de la lignée généalogique. Tout d'abord, dans l'éloge

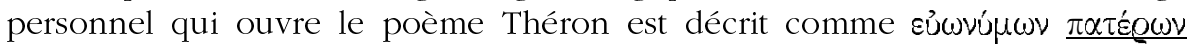

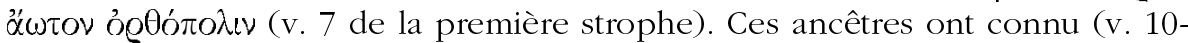
11) un aiciv $\mu o ́ \varrho \sigma \mu o c$ qui a apporté richesse et charis à leur vertus, qualifiées de yrńoı ( génos). Peu après, dans l'antistrophe (un exemple de parallélisme conceptuel), et en 'enjambement' avec la strophe suivante, on supplie Zeus de

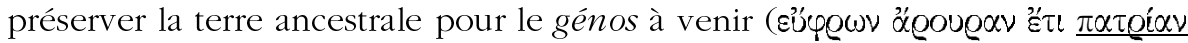

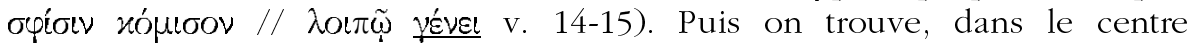
géométrique du poème, la mention de l'ascendance 'mythique' de Théron, en faisant référence au lignage d'CEdipe :

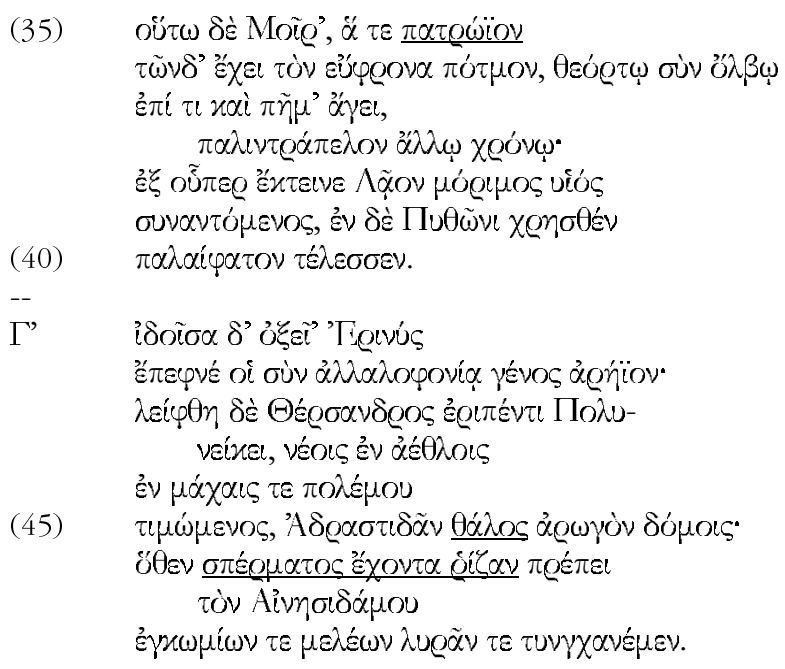

Ces références ont une valeur remarquable dans l'ensemble d'une ode dont l'architecture est parfaite et où s'accumulent les réflexions sur le temps, le destin, la justice, l'alternance de bonheur et de souffrance. Elle présente en outre deux sections qui ont en commun la démonstration des possibilités d'une existence joyeuse post mortem, même si celle-ci est abordée de façon

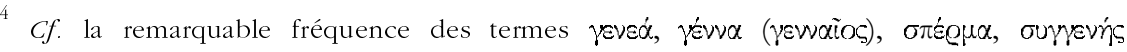

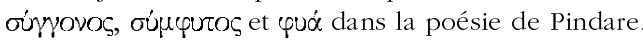


bien différente dans chaque cas. La victoire, ou plutôt les victoires, de Théron sont en parfait accord avec les qualités qui le caractérisent (notamment la justice), de telle façon qu'on pourrait considérer que sont conjurés les aspects négatifs du destin qui projettent leur ombre sur l'existence humaine en général et sur les Euménides en particulier, en ouvrant en même temps la possibilité d'un espoir eschatologique non moins positif.

Pour abréger, je me limite maintenant à quelques remarques qui soulignent le lien entre le passé de Thèbes, la réalité du moment présent et même l'existence dans l'au-delà, toujours autour de la question généalogiques.

(a) Ainsi, nous dit-on, les ancêtres occupèrent la terre de la Sicile, «après

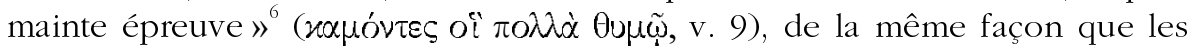

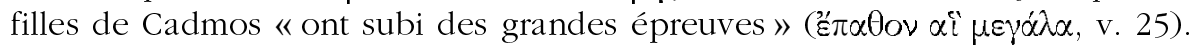
Une même yvóur pourrait servir pour les deux cas : «le poids de la peine

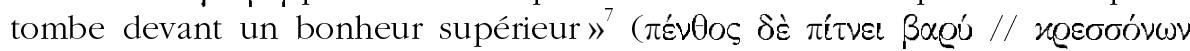

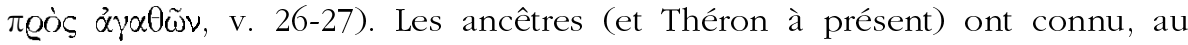

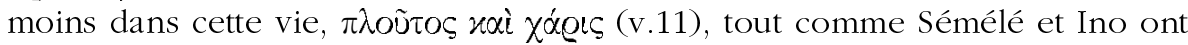
été récompensées par une vie impérissable à côté des dieux ou des Néréides.

(b) Justement après les vœux pour le génos du vainqueur (16) et les réflexions sur le temps et l'oubli (v. 19-20) la jvóun nous oriente vers la félicité dont jouit le fils d'Énésidame : "Par le noble effet de la joie, la peine

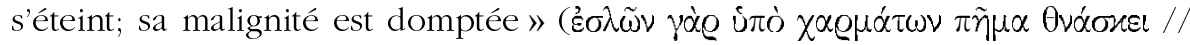
$\pi \alpha \lambda$ i $\gamma$ นotov $\delta \alpha \mu \alpha \sigma 0 \varepsilon \dot{v}$ v. 21-22). Or, dans le passage cité supra sur l'ascendance d'CEdipe, le terme $\pi \tilde{\eta} \mu \alpha$ reparait : la Parque peut apporter, à coté du bonheur, «en d'autres temps, par un retour inverse, quelque infortune»(40-42). Cependant, l'exemple d'CEdipe, qui illustre cette affirmation, fait contraste avec la réalité de Théron. CEdipe était prédestiné ( $\mu$ ógumoç vióc) et ne pouvait pas échapper à ce qui avait été prédit par l'oracle, de la même façon que l'Érinys «fit périr sa vaillante race» (46). Par contre, le nouveau rejeton de

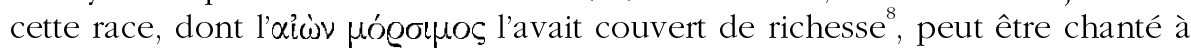
côté de son frère (v. 54). Tout montre bien que le destin du génos avait changé profondément avec Thersandros ${ }^{10}$.

${ }^{5}$ Il faut avoir bien présent à l'esprit que le triple niveau établi au commencement de l'ode entre dieux, héros et humains se poursuit tout au long du poème, qui nous parle des dieux (Zeus - Olympie ! -, Cronos, Athéna), des héroïnes de Thèbes (Sémélé, Ino) et des héros qui on connu une existence bienheureuse (Pélée, Cadmos, Achille), ainsi que d’un homme (Théron) qui, s'inscrivant dans une généalogie qui remonte à Cadmos et CEdipe, connaît une existence marquée par le bonheur et qui le fait digne de cette ascendance et d'un avenir bienheureux.

${ }^{6}$ Traduction d'A. Puech, Pindare (4 vols.), Paris, $1970^{6}$ (première édition 1922).

${ }^{7}$ Je modifie ici la traduction de PuEch (n. 6)

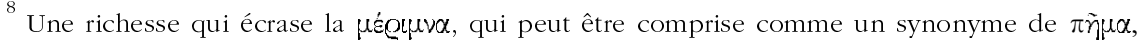
cf. v. 60-61

9 Cf. F.J. NiseTich, Pindar and Homer, Baltimore / London, Johns Hopkins University Press, 1989, p. $42-43$ pour ces idées.

${ }^{10}$ Cf. NisETICH, ibid., p. 63 : «Thersander, as we have seen, supplies the link between past and present, between Theron's Cadmean ancestors and Theron himself. Equally important, he marks the turn in Theron's ancestry from destructive to creative struggle, from strive within family 
(c) La mention de la racine qui naît de la semence de Thersandros et des Adrastides (v. 43-47) est placée entre les deux récits qui accomplissent la fonction du «mythe », celui du destin des filles de Cadmos et de la description qui mêle les éléments épiques et les doctrines orphiques. Par conséquent, j'oserais lui attribuer une sorte de fonction de "charnière » entre les traditions religieuses des ancêtres et les vivants, entre le dionysisme ancien des Thébains et la nouvelle version (d'un mysticisme complexe) de la Sicile du laudandus ${ }^{11}$.

Théron n'est pas le seul descendant de la lignée d'CEdipe chanté par Pindare. L'Isthmique III contient l'éloge de Mélissos de Thèbes ${ }^{12}$, dont l'ancêtre Cléonymos était un Labdacide du côté maternel. C'est à la fin de l'ode que le poète introduit de cette façon la référence à ce génos (v. 13-18b) :

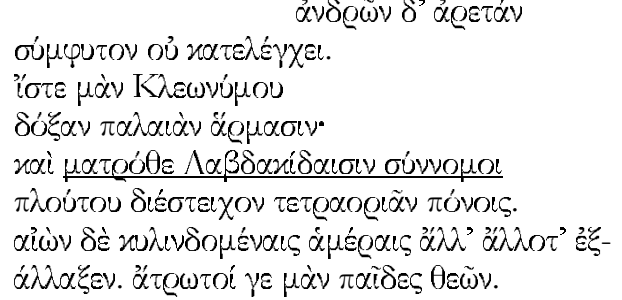

Comme l'a fait remarquer Privitera ${ }^{13}$, la yvíun s'adapte bien non seulement à la famille d'Edipe, mais aussi à celle du vainqueur, qui avait vu périr quatre de ses membres le même jour, à l'occasion, paraît-il (mais c'est une simple conjecture), de la bataille de Platées (479). Néanmoins, cette réflexion sur l'instabilité de la vie humaine par rapport à celle des dieux n'obscurcit pas le fait que le lien généalogique a été employé pour souligner les valeurs du

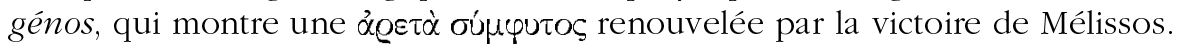

\section{Les pouvoirs d'un génos divin : Olympique 6 (472/468)}

L'éloge d'Hagésias, lieutenant de Hiéron, est fait non seulement en raison de sa victoire olympique, mais aussi en partant des vertus du génos, que le vainqueur maintient. C'est ainsi qu'il faut comprendre sa description comme

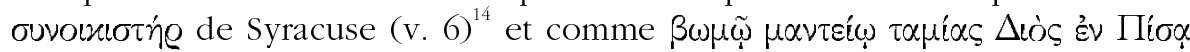
(v. 5), plutôt que d'en faire une simple exagération. Ce sont les vertus mantiques qui seront surtout soulignées dans le poème, par l'intermédiaire du

and city to athletic endeavor and heroism in war against foreign enemies. In him, finally, the two great traditions of epic poetry come together: the Theban, where he belongs as one of the Epigoni, and the Trojan, where he belongs as one of those who sailed with the Greeks against Troy. »

${ }^{11}$ L'élément en commun entre ces deux versions est qu'après les pathe il y a de l'espoir.

${ }^{12}$ En réalité, il venait de vaincre aux jeux Néméens une année après la victoire isthmique chantée dans Isthmique 4 , de 479.

${ }^{13}$ Pindaro. Le Istmiche, a cura di G. Aurelio Privitera, Milano, Mondadori, 1982 (Fondazione Lorenzo Valla), p. 171.

${ }^{14}$ Ses ancêtres avaient participé avec Archias à la colonisation de Syracuse. 
mythe, autant dans l'allusion rapide au personnage d'Amphiaraos ${ }^{15}$ que dans le développement du mythe central. Ce mythe établit le lien entre les niveaux divin, hérö̈que et humain : de l'union de Poseidon et Pitané naît Évadné, qui connaît l'amour d'Apollon et enfante Iamos, l'éponyme des Iamides, glorieux devins d'Olympie, et fondateur du lignage du vainqueur. Encore une fois le centre de la composition souligne les origines du génos. Quand Aipytos rentre de Delphes avec la réponse divine, il confirme l'avenir de cette race :

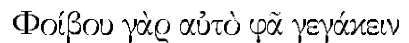

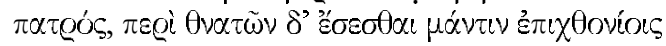

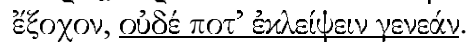

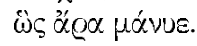

Le mythe sert aussi à établir un lien géographique entre le Peloponnèse et la Sicile, à deux niveaux, mythique et actuel, avec une forte implication de la personnalité du poète et de la fonction de la poésie. D'abord, le poète exhorte le cocher, Phintis, à atteler le char de mules qui vient de vaincre, avec lequel le poète va remonter au génos d'Hagésias, ce qui nous conduit dans le Péloponnèse $^{16}$. La métaphore unit l'épreuve sportive et l'art poétique. Puis, le poète mentionne un lien généalogique personnel avec le vainqueur, car la nymphe éponyme de Thèbes $(\Theta \dot{r} \beta \eta)$ était une fille du fleuve Asope et de Métope, nymphe éponyme du lac voisin de Stymphale, ville de la famille

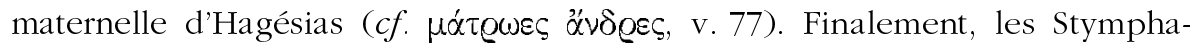
liens et les Syracusains vont partager la célébration de la victoire, car c'est de Stymphale que vient le cortège $(\varkappa \tilde{\omega} \mu o c)$, où le poète les a priés de chanter la

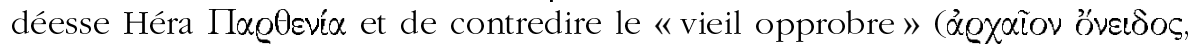

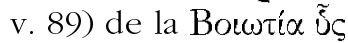

La perspective religieuse est très importante dans cette ode. Elle est aussi, dans une certaine mesure, en rapport avec la question généalogique. Dans la lignée directe d'Hagésias, on compte non seulement un, mais deux dieux, Poseidon et Apollon. Le dieu de la parole prophétique accorde à Iamos et à ses descendants les fonctions prophétiques à Olympie, domaine de Zeus, décrit comme $\beta \alpha \varrho u ́ \gamma \delta \delta o u \pi o s ~ \pi \alpha \tau \hat{n} \varrho$ (v. 81) dans un contexte (celui du quatrième épode, v. 77-84) qui introduit aussi la mention d'Hermès à propos du culte des ancêtres maternels en Arcadie, en tant que patron des jeux sportifs. L'exhortation aux 'compagnons' d'Énée (l'instructeur du chœur) nous rappelle le culte d'Héra Parthénie à Stymphale ${ }^{17}$. Puis, l'éloge de Syracuse,

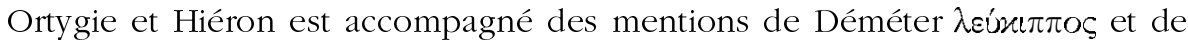
Zeus Aitvaíọ (v. 95-96). Finalement, le poète ferme l'ode avec une prière à

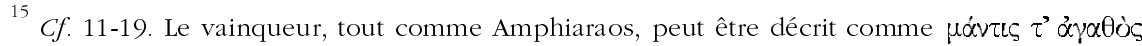

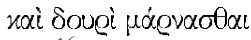

${ }^{16}$ Comme le fait remarquer PuEch (Olympiques, p. 82, n. 2) Pindare doit suivre une version différente de celle que raconte Pausanias (VIII, 4 et 16-17) et qui place la légende d'Aipytos dans le Nord de l'Arcadie, tandis que «la mention de l'Alphée suffit pour nous orienter vers une autre région ».

17 Paus., VIII, 22, 2.
} 


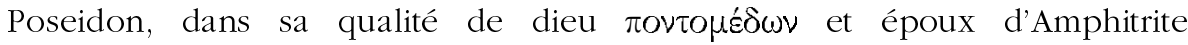

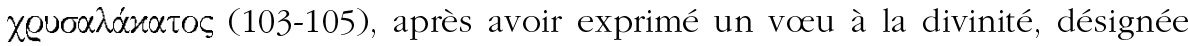
de façon plus générique comme $\theta \in o ́ c ̧ ~(101-2)$.

\section{Les alternatives généalogiques : Olympique 9 (468)}

Cette ode (en l'honneur du périodonique opountien Épharmoste) pose des problèmes d'interprétation importants, qui ont été traités très prudemment (et, à mon avis, résolus dans une bonne mesure) par Gerber ${ }^{18}$ dans son commentaire. Dans cet ensemble de problèmes, les questions généalogiques ne sont pas précisément les moindres. C'est à nouveau la partie centrale qui accueille la mention du lignage du vainqueur, Épharmoste, et des Opountiens. Le poète exprime son vœu de chanter la ville de Protogénie (vv. 41-42) et évoque comment Deucalion et Pyrrha avaient semé la «race de pierre» ( $\lambda$ í yóvov, v. 45), dont les gens ont reçu le nom éponyme de $\lambda \alpha o$. Puis il introduit une référence plus détaillée au déluge et à la descendance ultérieure dans les termes suivants (v. 49-66):

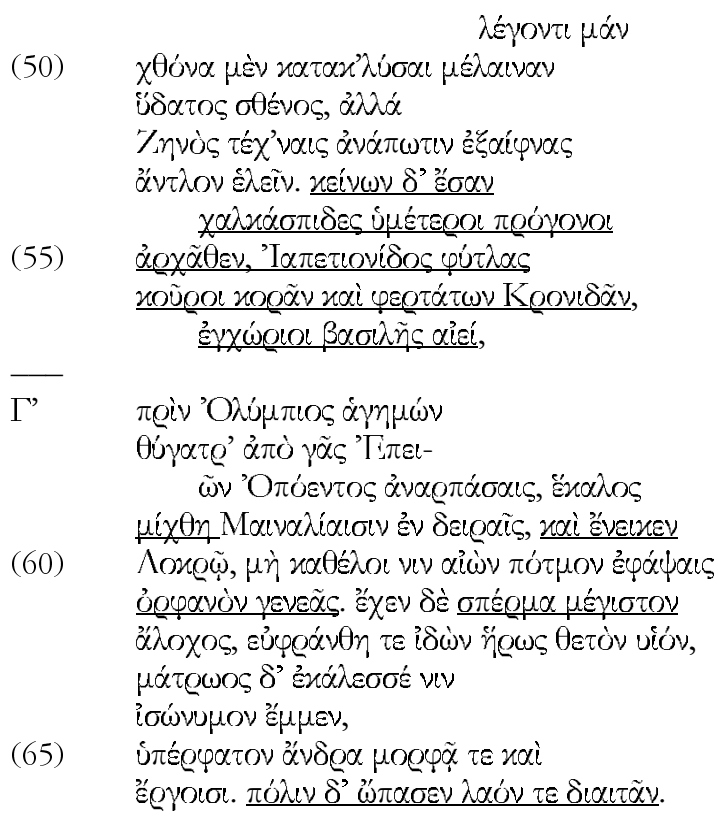

On est d'accord sur le principe que Pindare établit deux niveaux généalogiques. Le premier $(c f$. $\dot{\alpha} \varrho \chi \tilde{\alpha} \theta \varepsilon v)$ correspond aux générations nées immédiatement après le déluge. Le deuxième ( $c f$. $\pi$ @ív) au moment où Locre, sans descendance, adopte le fils de Zeus et de la fille d' Opous l'Épéen. Cependant l'identité des protagonistes de ces deux phases n'est pas évidente dans le texte pindarique. On ne saurait dire, par exemple, si les premiers reóyovol,

\footnotetext{
${ }^{18}$ D.E. GERBER, A Commentary on Pindar Olympian Nine, Stuttgart, Franz Steiner, 2002.
} 


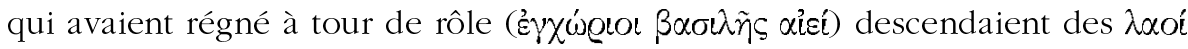
ou plutôt de Deucalion et Pyrrha. Le pronom xévvav (v. 54) étant ambigu, on ne dispose pas d'un critère décisif en principe, mais Miller ${ }^{19}$ a peut-être raison et le contexte fait penser plutôt au couple mythique, même si à la fin le résultat «does not differ significantly »" À l'appui de cette interprétation vient le fait que les vers suivants mentionnent la race de Japet (qui est le père de Prométhée et d'Épiméthée, les pères de Deucalion et de Pyrrha respectivement), ainsi que l'union de Protogénie et Zeus.

En effet, il est absolument nécessaire ${ }^{21}$ d'accepter que l'union de Zeus et

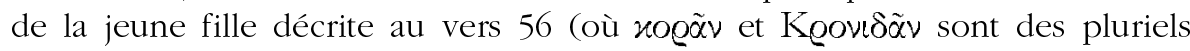
poétiques) n'est pas la même que celle des vers 57 sq. concernant la fille d'Opous $^{22}$. Ceci explique les efforts des scholies et des commentateurs pour éclairer cette version pindarique de la généalogie locrienne. "Pindar must mean that the royal line went back to a union between Zeus and Protogeneia », affirme raisonnablement Gerber ${ }^{23}$. Mais la difficulté est d'identifier alors qui est la mère d'Opous II, le fils de Zeus adopté par Locre. Déjà les scholies proposaient l'existence de deux Protogénies, une idée reprise parfois par la critique $^{24}$. Néanmoins, Huxley ${ }^{25}$ a ouvert une voie de solution intéressante : «The story of Opoeis I and Kaphya is the poet's innovation, and he candidly describes the pedigree as 'the flowers of newer hymns' (O. 9.48-49) ${ }^{26}$. Il observe que certaines sources ${ }^{27}$ mentionnent une fille du roi des Éléens, Opous, dont le nom était Kaçúx, éponyme de la ville de Káçuos, placée précisément au pied du mont Ménale. Cette Caphye (connue aussi comme Kabye) serait donc la candidate la plus apte comme mère d'Opous II. Par contre je n'oserais pas affirmer que la nouveauté proclamée par Pindare était exactement cette généalogie, car il serait un peu étrange que le poète ait voulu surprendre l'audience locale avec une modification si forte de ses traditions. Il ne faut pas perdre de vue qu'il introduit tout ce passage par l'expression renforcée $\lambda \dot{\varepsilon}$ yovtı $\mu \alpha \dot{\nu}$ (mais j'accepte que ceci peut être un simple recours rhétorique pour souligner la sensation d'objectivité).

Quoi qu'il en soit, le fait est que la mention généalogique a renforcé à nouveau l'éloge du vainqueur en établissant un lien divin et héroïque qui,

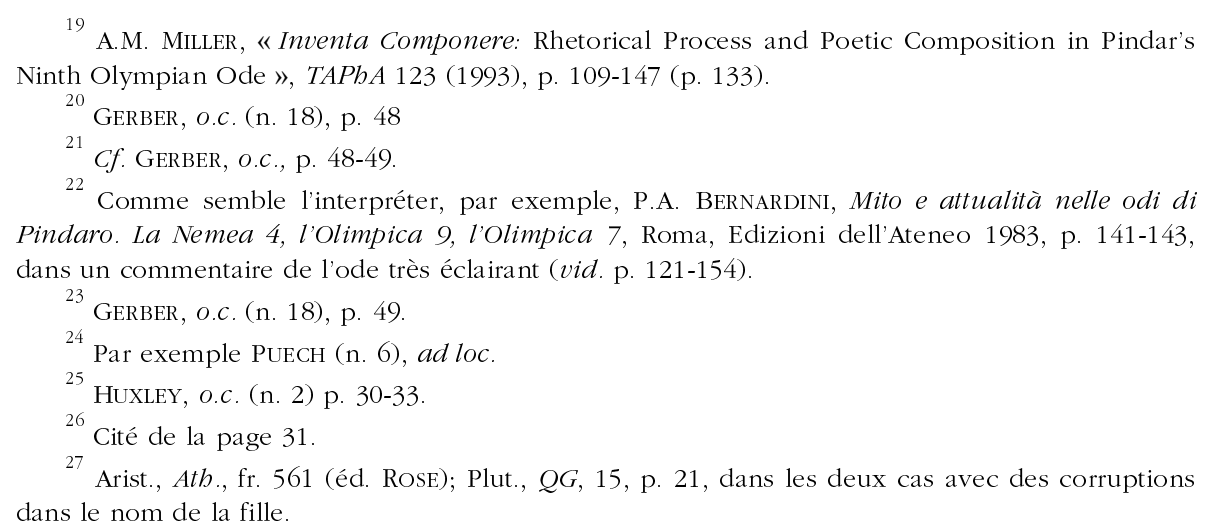


cette fois, fait remonter les origines du peuple chanté aux origines de l'humanité post-diluvienne et fait intervenir deux fois Zeus comme ancêtre (sans oublier les origines 'titaniques'). Cette glorieuse époque de Locre et Opous II (qui est à son tour un modèle de héros gouvernant) est sans doute un paradigme pour le présent ${ }^{28}$. C'est un temps où l'hospitalité mit les Locriens en rapport avec d'autres peuples et des héros épiques extraordinaires, comme Patrocle. On pourrait dire (comme il arrive souvent chez Pindare) que le présent victorieux d'Épharmostos était anticipé par le passé héroïque.

\section{La semence d'Héracles : Olympique 7 (464)}

Une autre ode exceptionnelle nous offre une nouvelle fois le motif généalogique comme élément important de l'éloge du vainqueur. Il s'agit cette fois du rhodien Diagoras, un autre périodonique fils de Damagétos, du génos des Ératides. La structure générale du poème est assez régulière (proème + actualité - mythe - actualité), tandis que le récit mythique présente des caractéristiques d'organisation assez particulières qui ont été soulignées souvent par la critique $e^{29}$. Le motif que nous intéresse est donc dans les parties initiale et finale, et n'est pas largement développé. Cependant, on doit mettre en relief le fait que le proème, avec l'image du banquet de noces où le père de l'épouse offre au nouveau gendre la coupe de vin, nous oriente vers un contexte social dans lequel les liens de famille sont substantiels et, comme l'a démontré Brown ${ }^{30}$, où l'appréciation des qualités du fiancé jouit d'une tradition poétique non moins consolidée. À côté de cette interprétation générale, on a proposé d'autres symbolismes implicites dans cette image. Ainsi, la coupe pleine de vin pourrait représenter la continuité de la famille à travers les générations ${ }^{31}$. La mention du gendre a été mise en rapport avec l'existence de ce même lien de famille entre le père de Diagoras, Damagète, et le héros messénien Aristomène ${ }^{32}$. Mais plus important encore, à mon avis, est le parallélisme qui s'établit entre l'image et la fonction poétique ${ }^{33}$.

Un éloge des valeurs de la famille du vainqueur était inévitable s'agissant des Ératides et, en l'occurrence, de Diagoras, un digne descendant d'Héraclès, même dans son aspect physique ( $\pi \varepsilon \lambda \hat{\lambda} \omega \dot{\varrho}$ เoৎ, v. 15$)^{34}$. Les origines de la famille

\footnotetext{
${ }^{28}$ Cf. BerNARDini, o.c. (n. 22), p. 143-148.

${ }^{29}$ Voir C. BARRIGÓn, «La 'logique' du récit mythique dans l'ode rhodienne de Pindare (Pind. O. VII) », Kernos 15 (2002), p. 41-52, avec la bibliographie antérieure.

${ }^{30} \mathrm{Ch}$. Brown, «The Bridegroom and the Athlete: The proem to Pindar's Seventh Olympian », in D.G. GERBer (ed.), Greek Poetry and Philosophy, Studies in Honour of Leonard Woodbury, Chico, Scholars Press, 1984, p. 37-50.

31 D.C. YOung, Three Odes of Pindar. A Literary Study of Pythian 11, Pythian 13 and Olympian 7, Leiden 1968 (Mnemosyne, suppl. 9), p. 74.

32 Voir B. LAvagnini, Da Mimnermo a Callimaco, Torino 1949, p. 89, repris par W.J. VERDENIUs, Commentaries on Pindar I: Olympian Odes 3, 7, 12, 14, Leiden, p. 44, n. 12.

${ }^{33}$ Cf. les remarques de Brown (o.c., n. 30) et de Verdenius (o.c., n. 32).

${ }^{34}$ Ses fils Acousilaos et Damagète furent aussi olympioniques en 448, le même jour, ce qui (d'après Aulu Gelle, III, 15) l'avait fait mourir de joie. Très célèbres ont été également son fils
} 
sont rappelées d'abord au commencement de la deuxième triade, en soulignant le oӨévoc comme caractéristique de cette race :

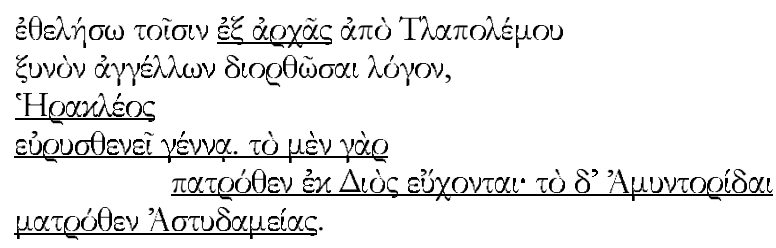

Zeus est le père d'Héraclès, père de Tlépolème, tandis qu'Amyntor, roi de Thessalie, était le père d'Astydamie, sa mère (Astyochée chez Homère, Il. II, 658, mais avec une autre origine ${ }^{35}$ ).

Puis, la fin de l'ode est une prière à Zeus, où on lui demande :

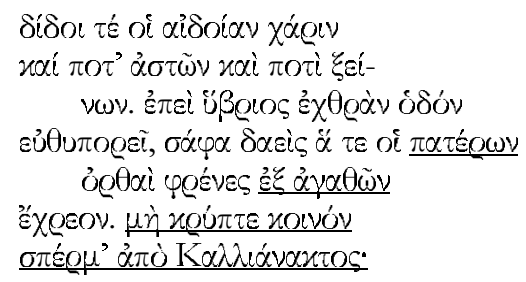

Cette fois, c'est la sagesse et la prudence qu'on fait apparaître en premier plan et on introduit un glorieux ancêtre, Callianax (qui devait évoquer certainement la gloire du génos) ${ }^{36}$. Ensuite la gnome ${ }^{37}$, avec l'image des vents qui changent subitement, ferme l'ode en laissant ouverte la réflexion sur la mutabilité des circonstances de l'existence, ce qui augmente la valeur des moments positifs et de gloire, comme sont ceux qu'ont vécus le vainqueur et sa famille.

La disposition des mentions généalogiques est donc pleinement stratégique, car elle construit un encadrement parfait dans un poème qui foisonne de récits mythiques fortement étiologiques et, dans le cas des origines de Rhodes et de sa descendance avec Poseidon, non moins "généalogique » (v. 54-76).

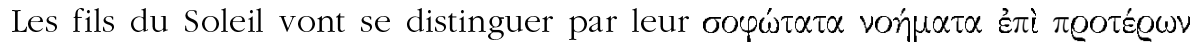

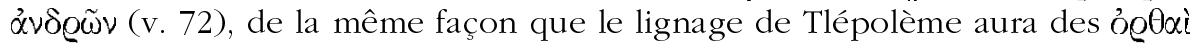
Y̧Q́vẹ (v. 91). Et c'est justement après cette étiologie de la fondation des trois villes de Rhodes qu'on passe au présent de l'île et au culte du fondateur Argien (v. 77-80), ce qui précède le catalogue des victoires de Diagoras et la nouvelle mention généalogique déjà citée.

Dorieus (trois fois périodonique; vainqueur olympique en 432, 428, 424) et ses petits-enfants Peisirode et Euclès (vainqueurs olympiques en 404). Voir les anecdotes recueillies par Pausanias (VI, 7, 2; V, 6, 7-9) sur cette famille.

${ }^{35}$ Cf. PUECH (n. 6), p. 95, n. 4.

36 D'après les scholies il est un ancêtre de Diagoras; par contre, J. Poulloux, « Callianax, gendre de Diagoras de Rhodes. À propos de la VII ${ }^{\mathrm{e}}$ Olympique de Pindare », RPh 44 (1970), p. 206-214, fait l'hypothèse qu'il s'agit de son gendre.

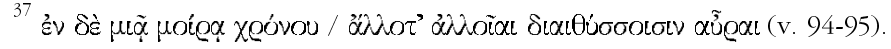




\section{Reconstruction du passé, niveaux chronologiques et archi- tecture d'un poème : Pytbiques 4 et 5 (462)}

Ces deux poèmes commémorent une même victoire à la course des chars d'Arcésilas IV de Cyrène, un descendant (à la huitième génération) du premier colonisateur de la ville, Aristote-Battos. La composition de deux odes pour un même événement n'est pas unique chez Pindare, mais c'est assez exceptionnel et oblige à analyser soigneusement les différences entre les deux compositions. Raisonnablement on peut juger que la vraie épinicie serait plutôt la cinquième Pythique, tandis que la quatrième (la plus longue des compositions pindariques) montre des particularités qui laissent entrevoir un ambitieux programme encomiastique qui n'est pas exempt d'une composante politique importante ${ }^{38}$. Ces caractéristiques spéciales deviennent assez évidentes dans l'organisation structurale. C. Calame nous a fait le cadeau d'une belle analyse des questions sémio-narratives qui affectent cette ode ${ }^{39}$ et il serait superflu de revenir sur les multiples aspects qu'il a traités en profondeur. Je me bornerai à reprendre la question des niveaux chronologiques, en soulignant les points qui sont en rapport avec le motif généalogique.

Je suis pleinement d'accord avec Calame quand il détecte cinq niveaux temporels dans ce poème ${ }^{40}$, mais je vais me permettre de modifier un peu la description de ces couches. En séquence linéaire les points de repère seraient les suivants: (1) Euphamos reçoit la motte de terre que lui donne Eurypyle, qui lui annonce une future fondation. (2) Prophétie de Médée pendant l'expédition des Argonautes. Cette prophétie a à son tour des éléments «en arrière » et «en avant», car Médée non seulement annonce des événements futurs (3), mais aussi rappelle l'épisode d'Euphamos et raconte ce qui aurait dû se passer si la motte de terre était arrivée (comme prévu) à la bouche de l'Hadès (fondation manquée à la quatrième génération). (3) Union d'Euphamos (et des Argonautes) avec les Lemniennes au retour de l'expédition en Cholchide. (4) De cette union, après dix-sept générations, naîtra Battos, qui colonisera Cyrène en partant de l'île de Théra. (5) Temps de l'exécution de l'ode, génération d'Arcésilas.

Or, l'organisation du récit devient assez complexe (mais non privée de symétrie). Dans cette structure la parole prophétique devient un instrument essentiel (en réalité les trois odes cyrénéennes sont vraiment «apolliniennes », en fonction du culte local). Qu'on me permette de reprendre ce que j'avais dit dans mon exposé du colloque de 1989: "Nous sommes donc en face d'une sorte de 'canevas' prophétique, dont l'ensemble est encadré par les oracles d'Apollon à Battos (4 sq., 53 sq., 259 sq.) en alternance avec les

\footnotetext{
${ }^{38}$ Que je ne vais pas traiter maintenant, car je vais me limiter aux questions en rapport avec le sujet de cette étude.

39 Ainsi que l'ensemble des trois odes cyrénéennes : o.c. (n. 3), Mythe et bistoire..

${ }^{40}$ O.c., p. 73: T 1: temps de l'exécution de l'ode (Arcésilas IV), T 2: temps de l'acte fondateur (Battos), T 3: temps du programme non réalisé (Danaens), T 4: temps de la prophétie (Médée), T 5: temps de la manipulation (Euphémos).
} 
références à l'actualité (1 sq., 64 sq., 263 sq. et, plus concrètement, 270 sq.). Enfermé dans ce premier niveau se trouve un deuxième encadrement structurel qui a comme points de repère la prophétie sur l'île de Théra (13 sq.) et l'évocation de son accomplissement (258)»" Au centre de ces anneaux se trouve le long récit mythique. Le résultat est la présentation des couches temporelles qu'on vient d'exposer dans l'ordre suivant : $5-4-2$ (avec mention de 1 et de 'l'occasion perdue', ainsi que l'annonce de 3) - $4-5$ - MYTHE - 3-4 - 5 (avec le prolongement de la défense de Damophile).

On aperçoit donc un profond intérêt pour souligner le rapport entre le héros fondateur Battos (sans oublier à son tour ses origines) et le présent de la famille royale locale. Comme je viens de le dire, cela se fait par trois fois, avec une intensité progressive :

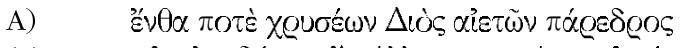

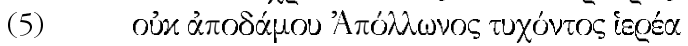

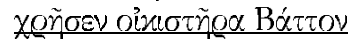

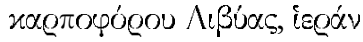

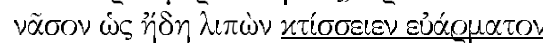

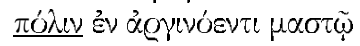
B) $\quad \tilde{\omega} \mu \alpha \dot{x} x \alpha Q$ vi̇̀ Пo

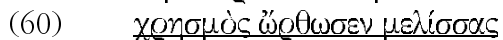

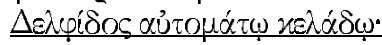

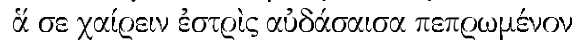

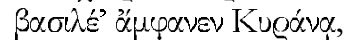

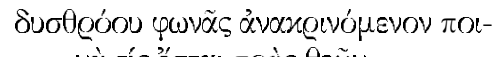

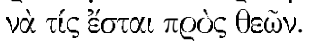
$\tilde{\hat{n}} \mu \alpha \lambda \alpha \delta \dot{\varepsilon} \mu \varepsilon \tau \dot{\alpha} \alpha \alpha i v \tilde{u} v$

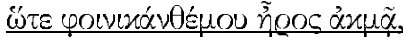

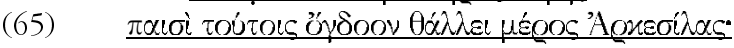

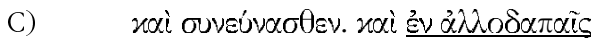

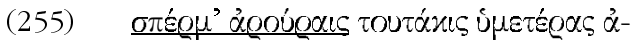

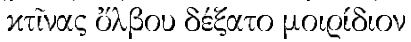

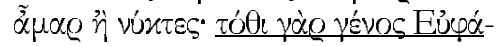

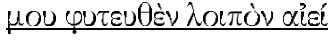

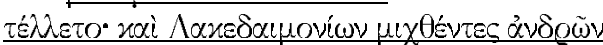

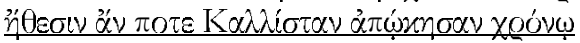

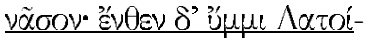

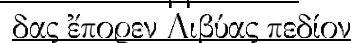

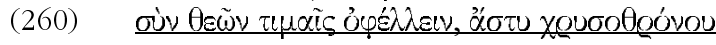
Sıavépeiv Aeion Kugávas

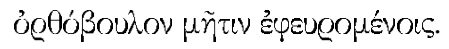

\footnotetext{
${ }^{41}$ E. SUÁREZ DE LA TORRE, «Parole de poète, parole de prophète : les oracles et la mantique chez Pindare », Kernos 3 (1990), p. 347-358, citation p. 352.
} 
Remarquons, en effet, la gradation depuis la première mention simple de la fondation (A) jusqu'à l'expansion de la dernière à propos des vertus politiques de la famille (C), en passant par le lien entre Battos et Arcésilas, exprimé par l'image végétale (B). Dans le dernier passage cité on introduit aussi le motif de la protection divine et du caractère sacré de la fondation ( $c f$. en trois lignes les mentions d'Apollon, des dieux, du caractère sacré de la ville et de Cyrène « au trône d'or ») ${ }^{42}$.

Cette combinaison de liens généalogiques héroïques et mise en relief des éléments religieux et de la sauvegarde divine est un trait partagé avec cette ode par la Pythique 5 dont la partie centrale, normalement réservée au mythe, est occupée par les mentions de la colonisation et des traditions religieuses et cultuelles locales, étroitement liées les unes aux autres. Par trois fois on trouve dans cette ode (d'un ton encomiastique considérable) le motif généalogique et, comme dans l'ode précédente, avec une sorte de gradation dans ses dimensions. D'abord dans l'éloge initial, dans une référence au mélange des vertus de la race et les qualités personnelles (avec une certaine difficulté syntaxique et dans un contexte qui proclame l'ổ $\lambda \beta \emptyset$ du vainqueur, v. 17-19):

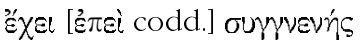

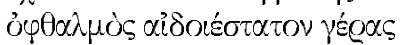

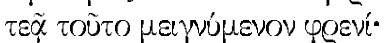

Puis, dans la première référence au fondateur de la ville (vv. 55 ss.), Battos, dont on rappelle sa fonction de protecteur de la ville et des concitoyens :

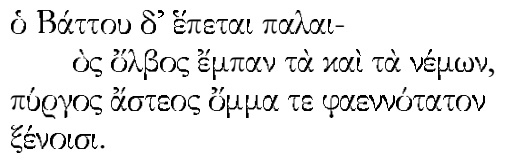

Cette mention est suivie du souvenir de son arrivée sur le territoire de Cyrène, ainsi que de la protection que lui avait octroyée Apollon, qui reçoit ici la dénomination d'ả $\varrho \chi \alpha y e ́ \tau \alpha \subseteq$ (v. 60). Finalement, après une belle énumération des pouvoirs d'Apollon, le poète évoque en détail les origines de la race colonisatrice du territoire. C'est un long passage, qui évoque la migration dorienne à Théra (l'île qui reçoit le nom de son colonisateur, Théras) ${ }^{43}$, l'occupation ancienne du territoire Libyen par des Troyens, l'arrivée de l'expédition d'Aristote-Battos (v. 69-95) et le culte héroïque qu'il reçoit dans l'agora de la ville. Dans la référence aux Égides (v. 73-76) une partie de la

42 Cf. Pindaro. Le Pitiche, a cura di B. Gentili, P.A. Bernardini, E. Cingano, P. Giannini, Milano, Mondadori, 1995 (Fondazione Lorenzo Valla), p. 498 (à propos des v. 254-262) : « qui è sottolineata in particolare l'origine remota della famiglia, mentre lì è dato maggiore spazio alle vicende dell'oracolo di Delfi (...). La ripresa è indicio della particolare rilevanza di questi fatti per l'intento celebrativo dell'ode. »"

${ }^{43}$ Auparavant son nom était Kallista. 
critique a vu une allusion aux ancêtres du poète, tandis que d'autres considèrent qu'on doit appliquer ces affirmations au chour de Cyréneens (v. 72-93) ${ }^{44}$ :

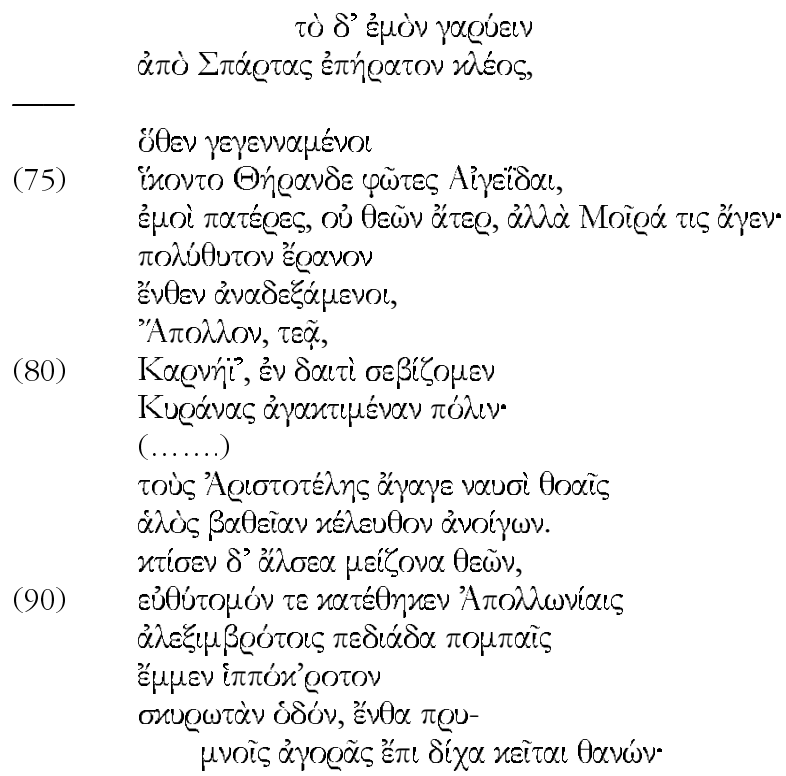

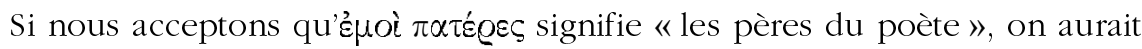
un cas proche de celui de l'Olympique 6 (et par conséquent normal dans l'usage pindarique), sinon on aurait une mention généalogique pure. En tout cas, on doit insister à nouveau sur le lien qu'on établit entre la fondation et le moment présent, un lien construit sur les traditions religieuses: le culte d'Apollon Karnéios et celui de Battos. Le poète n'oublie non plus les autres

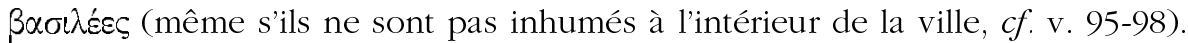
Une fois établie la fusion entre passé et présent on passe à nouveau à l'éloge d'Arcésilas, qui fermera le poème.

\section{La généalogie dans l'enkomion d'un prytane : Néméenne 11}

Ode de date inconnue, elle a été ajoutée à la fin de l'ancienne édition qui se terminait par les Néméennes, probablement à cause de l'extension de l'énumération des victoires agonistiques d'Aristagoras de Ténédos. Cette ville avait comme institution politique fondamentale un collège de prytanes dont Aristagoras devait faire partie l'année de la composition. On comprend donc parfaitement le ton de l'ode, qui combine les appels à la réflexion sur les vicissitudes de l'existence et même un memento mori avec des traits plus propres d'un enkomion. La mention généalogique associe parfaitement les deux aspects, car, d'un côté, elle illustre bien comment les moments de succès et de gloire ne forment pas un continuum dans la vie de l'homme

\footnotetext{
${ }^{44}$ Je renvoie au commentaire du passage dans Pindaro. Le Pitiche, o.c. (n. 42$)$.
} 
mais, de l'autre, elle explique la possibilité de connaître des moments de rayonnement des vertus héréditaires. Cette fois, la généalogie mérite d'être chantée, car elle suppose la fusion de deux lignages mythiques importants ( $\mathrm{v}$. 33-38) :

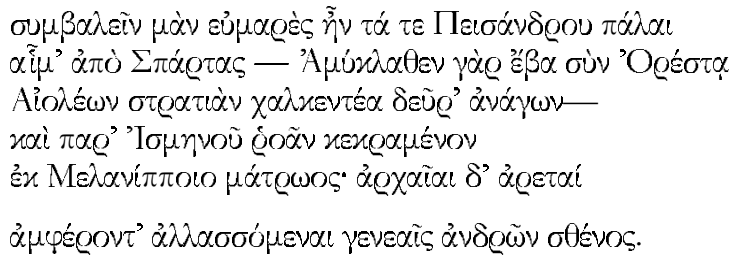

Pindare attribue à Pisandre et à Oreste une colonisation que d'autres sources assignent à Penthilos ${ }^{45}$. Aristagoras porte dans son sang (en vertu de la lignée maternelle) les vertus autant de ces héros que de Mélanippe.

\section{Conclusions}

Neuf épinicies pindariques introduisent le motif généalogique. Parmi elles se trouvent quelques-unes des plus élaborées et complexes du point de vue de l'organisation interne. L'analyse de ces odes nous permet d'établir les fonctions et traits suivants de la mention généalogique.

Elle renforce la perspective temporelle et la dimension historique qui explique le moment de gloire du présent comme une conséquence naturelle des origines et de la trajectoire de la famille du vainqueur. Le «ol@ós qui fait briller la victoire devient possible parce que les vertus du lignage peuvent apparaître à un moment donné, malgré les multiples vicissitudes de l'existence du génos. En effet, ses membres émanent d'une semence divine ou hérö̈que dont la force est par-dessus ces vicissitudes.

Un élément important dans cette perspective temporelle (et dans l'établissement de liens entre le présent et le passé), c'est le facteur religieux, dont la présence peut présenter des traits variables. Le culte du dieu du lignage ou du héros colonisateur et fondateur du territoire et du génos soutient la mémoire des origines, ce qui est revitalisé par l'interprétation publique de l'ode. Le cas de l'Olympique 2 est exceptionnel, dans le sens où elle ouvre aussi à une dimension religieuse eschatologique complexe qui semblerait pouvoir conjurer (avec l'espoir d'immortalité sous certaines conditions) les aspects négatifs des oscillations du bonheur dans l'existence humaine.

La mention généalogique renforce souvent le souvenir du fait colonisateur et introduit donc également une dimension spatiale qui s'ajoute à celles du temps et de l'histoire. De cette façon, on établit le lien entre le continent et les îles (Sicile, Rhodes) ou entre deux continents (Europe, "Libye ») ou entre plusieurs territoires (Locride, Arcadie, etc.). La généalogie articule donc une

\footnotetext{
${ }^{45}$ Strabon, IX, 2, 5 (C402).
} 
sorte de conscience d'unité par-dessus l'espace et le temps qui est mise en relief par la remémoration poétique.

Le poète se sert de la mention généalogique pour organiser la stratégie de l'organisation du poème. (a) Elle peut occuper une position centrale (en fait le centre du poème, $c f$. Olympiques 2, 6 et 9), soit en faisant partie de l'éloge direct du vainqueur soit, comme dans l'Olympique 6 (en position très emphatique, renforcée cette fois par une prédiction apollinienne) et la Pythique 5, incorporée à la narration mythique ou au récit qui remplit cette fonction ${ }^{46}$. (b) Dans d'autres cas (Olympique 7, Pythique 4), elle apparaît au commencement et à la fin, dans les parties qui encadrent le récit mythique et peut présenter une sorte de gradation qui renforce en climax les liens entre le passé et le présent. (c) Dans les odes plus courtes (Isthmique 3, Néménne 11) elle apparaît à la fin, mêlée à l'éloge des vertus du laudandus.

En somme, elle est un des recours les plus efficaces pour renforcer la fonction encomiastique de l'épinicie et devient presque un passage obligé s'agissant de personnages appartenant à des familles très puissantes étant parfois eux-mêmes de vrais 'héros vivants' du monde sportif (même des périodoniques).

Emilio SuÁreZ DE LA TORRE

Universidad de Valladolid

E-mail : esuarez@vasertel.es

\footnotetext{
${ }^{46}$ Dans la Pythique 5, la colonisation occupe la position centrale.
} 